\title{
Bovine Tuberculosis and Badger Culling in England: A Utilitarian Analysis of Policy Options
}

\author{
Steven P. McCulloch ${ }^{1} \cdot$ Michael J. Reiss $^{2}$
}

Accepted: 30 July 2017/Published online: 18 August 2017

(C) The Author(s) 2017. This article is an open access publication

\begin{abstract}
Bovine tuberculosis (bovine TB) is an important animal health policy issue in Britain, which impacts farmers, the public, domestic farmed cattle and the wild badger population. The Westminster government's badger culling policy in England, which began in 2013, has caused considerable controversy. This is in part because the Independent Scientific Group advised against culling, based on the Randomised Badger Culling Trial. Those opposed to badger culling support more stringent cattle-based measures and the vaccination of badgers. This paper argues for ethical analysis of public policy options which impact sentient species. It provides a summary Animal Welfare Impact Assessment of (1) a do-nothing approach, (2) badger culling, and (3) badger vaccination. A utilitarian analysis is then applied to these policy options considering human wellbeing and animal welfare. The analysis compares a badger culling policy that achieves a $19 \%$ reduction in bovine TB incidence, a badger vaccination model achieving a $12.5 \%$ reduction, and a donothing approach. Policy options are assessed over 9 years and a longer timeframe, and uncertainty is considered. The analysis finds that non-culling approaches, particularly badger vaccination, result in greater total utility, compared to badger culling. Badger culling causes 30\% reduction in the badger population in England as well as substantial harms due to the culling process. Culling is opposed by public opinion and is associated with considerable risks and uncertainty. In contrast, nonculling approaches, such as cattle-based measures and badger vaccination, are supported by public opinion and are not associated with such risks.
\end{abstract}

Steven P. McCulloch

Steven.McCulloch@winchester.ac.uk

1 University of Winchester, Sparkford Road, Winchester SO22 4NR, UK

2 UCL Institute of Education, University College London, 20 Bedford Way, London WC1H 0AL, UK 
Keywords Animal Welfare Impact Assessment (AWIA) · Badger culling ·

Badger vaccination · Bovine tuberculosis - Government policy · Utilitarianism

\section{Introduction}

Bovine tuberculosis (bovine TB) is an important and highly controversial policy issue in Britain. It is controversial particularly because of the role of the badger, Meles meles, which is a wildlife reservoir of infection. In England, the government has followed a badger culling policy as part of a package of measures to control bovine TB in cattle. However, many claim that badgers should not be culled and some argue for a vaccination policy instead. This paper provides a utilitarian ethical analysis of bovine TB badger control policy options in England. First, a brief overview of bovine TB and badger control policy is provided. Secondly, the paper argues for the inclusion of ethical analysis to inform animal health and welfare policy, such as bovine TB. Thirdly, a summary Animal Welfare Impact Assessment (AWIA) of badger control policy options is provided. Fourthly, the paper provides a brief overview of utilitarian moral theory and analyses bovine TB and badger control policy in the context of a utilitarian framework.

\section{Bovine TB and Badger Control Policy in England}

Bovine TB has been described by government as the most important animal health policy issue in England (Defra 2011c, 2014b). The policy issue is highly controversial, principally for two reasons. First, the 2010-2015 Coalition government and the 2015-present Conservative government have followed a badger culling programme. The badger is a cherished wildlife species with an important role in British culture and literature. This has contributed to substantial public opposition against badger culling (Defra 2006; HM Gov 2013). Secondly, the scientific evidence on badger culling is highly contested. For instance, the Independent Scientific Group (ISG) recommended against badger culling based on the findings of the Randomised Badger Culling Trial (RBCT) (Bourne et al. 2007). However, the subsequent King review claimed that badger culling could contribute to bovine TB control in cattle (King 2007).

Pilot badger culls commenced in west Somerset and west Gloucestershire in 2013. The culls did not meet the efficacy or humaneness criteria set by the government. Based on RBCT data, the culling companies had a target of culling $70 \%$ of the badger population over 6 weeks in licence areas. However, in Somerset less than $48 \%$ and in Gloucestershire less than $39 \%$ were culled (IEP 2014). In terms of humaneness, the government set a target of less than $5 \%$ of badgers taking over 5 min to die. However, the Independent Expert Panel (IEP), which monitored the pilot culls, reported that 7.4-22.8\% of badgers took over 5 min to die after being shot (IEP 2014). The shortcomings of the pilot culls led the government to postpone its planned 2014 roll out across high bovine TB incidence areas. The culls in Somerset and Gloucestershire have continued annually from 2013-2016. The 
government later announced a new culling area, north Dorset, to commence culling in 2015, and seven new areas commenced culling in 2016 (Defra 2016c).

The analysis in this paper concerns bovine TB and badger control policy options in England. Scotland was granted Officially Bovine TB Free (OTF) status in 2009. Wales has tightened cattle-based measures, moving to annual testing on a national basis. It has also followed a badger vaccination policy in its 'Intensive Action Area' in Pembrokeshire. From 2008 to 2015, the bovine TB incidence in Wales decreased by around 50\% (APHA 2015, pp. 6, 18). More recently, in part due to a global shortage of the BCG vaccine, badger vaccination has been halted in Wales and the government has agreed to targeted culling of badgers on some chronically-infected farms (Messenger 2016; Welsh Government 2017). The science, policy and politics of badger culling are discussed further in McCulloch and Reiss (2017).

\section{The Justification for Ethical Analysis of Bovine TB and Badger Control Policy}

Natural England advice to Defra prior to the 2013 pilot culls estimated that $30-50$ badgers would be killed for each bovine TB herd breakdown prevented if culling were successfully implemented. The level of culling proposed would cause a 14-27\% reduction of the badger population in England, and a 25-54\% reduction in the South West and West Midlands (Natural England 2011, p. 5). Badger culling is clearly an ethical issue: Policy on badger control should not, and indeed cannot, be based exclusively on the scientific evidence base (e.g. the RBCT), economics (e.g. a cost-benefit analysis) nor public opinion (e.g. a Defra 2006 public consultation). Ultimately, policy on badger control is a moral issue that must be analysed in the context of the following question:

Ethically, what is the right, or most justifiable policy on badger control, considering impacts on all morally relevant affected groups?

The natural science evidence base, economic tools and public opinion should all inform policy. However, given that cattle and badgers are sentient creatures, government policy should be appraised in the context of the above question. This entails conducting the following prior to decision making:

1. An Animal Welfare Impact Assessment (AWIA) to assess the impacts of policy options on cattle and badger populations, and

2. Robust ethical analysis, informed by the empirical evidence base, of badger control policy options.

The exclusion of either AWIA or independent ethical analysis, or indeed both, leads to the exclusion of the interests of animals in government policy. The public has a legitimate moral concern about how public policy impacts sentient animals. Therefore, the exclusion of 1 and/or 2 leads to the exclusion of legitimate values held by the democratic public about how society treats animals. The following 
section provides a summary of AWIA applied to bovine TB and badger control policy options.

\section{Animal Welfare Impact Assessment (AWIA) of Bovine TB and Badger Control Policy Options}

We have argued for the inclusion of mandatory Animal Welfare Impact Assessment (AWIA) for all public policy that affects sentient nonhuman species (McCulloch 2015; McCulloch and Reiss 2017). AWIA is a robust mechanism to assess how policy options impact the interests of sentient species. Briefly, AWIA is conducted in three stages:

1. Harms and benefits list.

2. Species description.

3. AWIA analysis.

The three stages of AWIA are conducted for each policy option under consideration for all sentient species significantly impacted. The AWIA analysis stage assesses impacts in terms of life and killing impacts, and positive and negative welfare impacts. In McCulloch and Reiss (2017) we have applied AWIA to the three badger control policy options of (1) do nothing, (2) badger culling, and (3) badger vaccination. The do-nothing policy option refers to badger control alone. Hence, bovine TB policy would be based on cattle-based measures such as tuberculin testing and slaughter of reactors, movement restrictions and inspection of carcasses at slaughterhouses. For the purpose of utilitarian analysis, the following sections include the species descriptions for cattle and badgers and summary AWIA analyses for the three policy options.

\section{Animal Welfare Impact Assessment: Species Descriptions}

The species description for the cow, Ox borus, is found in Table 1, and that for the badger, Meles meles, in Table 2. The species descriptions include data that may influence ethical analysis of policy options.

\section{Animal Welfare Impact Assessment: Summary AWIA Analyses}

Tables 3 (cattle population) and 4 (badger population) include summary AWIA analyses for the three policy options. A complete AWIA includes positive and negative welfare impacts in terms of duration (short, medium, long) and intensity (mild, moderate, strong) (McCulloch and Reiss 2017). The values in Tables 3 and 4 represent the total positive and negative welfare impacts on cattle and badgers respectively. Footnotes to Tables 3 and 4 show how these total welfare impacts, as well as life and killing impacts, are calculated, including references to source material where appropriate. 
Table 1 AWIA species description-cow

\begin{tabular}{|c|c|c|}
\hline Category & Description & Comments \\
\hline Species name & Bos taurus & \\
\hline Common name & Cow/bull/oxen & \\
\hline Population number & $\begin{array}{l}\text { 5.308 million total cattle and calves in England } \\
\text { (Defra 2014a) } \\
\text { 9.7 million total cattle and calves in UK (Defra } \\
2013 \text { ) } \\
\text { 1.78 million dairy herd in UK (DairyCo 2014a) }\end{array}$ & \\
\hline Global population & $\begin{array}{l}1.4 \text { billion (FAO 2010, p. 46) } \\
260 \text { million dairy cows-FAO } 2011 \text { in DairyCo } \\
(2014 \text { b) }\end{array}$ & \\
\hline Domesticated/wild & Domesticated in England & \\
\hline Human use or relation & Food (milk and beef production) & \\
\hline $\begin{array}{l}\text { Natural lifespan } \\
\text { (longevity) }\end{array}$ & $\begin{array}{l}20-25 \text { years } \\
12 \text { years or older (dairy cow) (FAWC 2009b, p. 2) }\end{array}$ & \\
\hline Normal lifespan (average) & $\begin{array}{l}6 \text { years (FAWC 2009b, p. 9) } \\
1-2 \text { years }\end{array}$ & $\begin{array}{l}\text { Figure for dairy cow } \\
\text { Figure for beef } \\
\text { animal }\end{array}$ \\
\hline Population status & Not endangered & \\
\hline Other important features & None & \\
\hline
\end{tabular}

The data in the AWIA analyses and the basis of the utilitarian analysis of badger control policy are based on the following baseline assumptions. First, the badger culling policy achieves a $19 \%$ reduction in bovine TB incidence. This is achieved by culling $70 \%$ of badgers in 33 lots of $350 \mathrm{~km}^{2}$ cull areas. Badgers are culled annually for 4 years, and the reduction in bovine TB incidence is measured over 9 years. Hence, the policy is to cull badgers annually for 4 years to achieve a reduction in bovine TB of $19 \%$ over 9 years. It is important to note here that the target of a $19 \%$ reduction in bovine TB is likely to overestimate the benefits of badger culling. Natural England advice to Defra prior to the 2013 pilot culls stated that the $19 \%$ figure is based on the 'Donnelly model', which assumes badger culling for 5 years plus a 4-year post-culling period (Jenkins et al. 2010). However, the badger culling licences are issued by Natural England for (a minimum of) 4 years of culling and a 5-year post-culling period (Natural England 2011). Finally, badger culling is conducted by using $50 \%$ controlled/free-shooting and $50 \%$ cage-trapping and shooting. ${ }^{1}$

The badger vaccination option is based on the following assumptions: first, annual BadgerBCG vaccination reduces the transmission of $M$. bovis from vaccinated badgers to cattle by $50 \%$. There is limited direct data for the efficacy of BadgerBCG. However, Chambers et al. have shown that BadgerBCG vaccination

\footnotetext{
1 The Defra summary report of the 2016 culls reveals that $55 \%$ of badgers were culled using controlled/ free-shooting and 45\% using cage-trap and shooting (Defra 2016d).
} 
Table 2 AWIA species description-badger

\begin{tabular}{|c|c|c|}
\hline Category & Description & Comments \\
\hline Species name & Meles meles & \\
\hline Common name & European badger & \\
\hline \multirow[t]{4}{*}{ Population number } & 220,000 (England) (Natural England 2011) & \multirow{4}{*}{$\begin{array}{l}\text { Estimation by } \\
\text { natural England }\end{array}$} \\
\hline & $\begin{array}{l}\text { 190,000 (England) (Battersby and Tracking } \\
\text { Mammals Partnership 2005, p. 84) }\end{array}$ & \\
\hline & $\begin{array}{l}\text { 300,000 (Great Britain) (British Wildlife Centre } \\
\text { 2012) }\end{array}$ & \\
\hline & $\begin{array}{l}50,241 \pm 4327 \text { badger setts (Great Britain) (Wilson } \\
\text { et al. } 1997, \text { p. } 7 \text { ) }\end{array}$ & \\
\hline Global population & No data & \\
\hline Domesticated/wild & Wild & \\
\hline Human use or relation & $\begin{array}{l}\text { Wildlife-aesthetic. Carnivorous species. Diet } \\
\text { includes earthworms and small mammals }\end{array}$ & \\
\hline \multirow[t]{2}{*}{ Natural lifespan (longevity) } & 3-5 years (Godwin-Pearson 2012, p. 19) & \\
\hline & 6 (average)—14 (high) years (Wang 2011) & \\
\hline Normal lifespan (average) & 16 years captivity (Wang 2011) & $\begin{array}{l}\text { Not normally } \\
\text { captive }\end{array}$ \\
\hline \multirow[t]{4}{*}{ Population status } & IUCN classification Least Concern & \\
\hline & Listed on Appendix III of Bern Convention & \\
\hline & $\begin{array}{l}\text { Listed on Schedule } 6 \text { of the UK Wildlife and } \\
\text { Countryside Act } 1981\end{array}$ & \\
\hline & Listed under UK Protection of Badgers Act 1992 & \\
\hline \multirow[t]{3}{*}{ Other important features } & Largest UK land wildlife species & \\
\hline & $\begin{array}{l}\text { Up to } 50,000 \text { killed annually by road traffic } \\
\text { accidents (WildCRU 2015) }\end{array}$ & \\
\hline & $\begin{array}{l}\text { Up to } 10,000 \text { killed annually by illegal baiting and } \\
\text { digging (WildCRU 2015) }\end{array}$ & \\
\hline
\end{tabular}

reduces seroprevalence in badgers by $73.8 \%$ (Chambers et al. 2011). Hence, the assumption of BadgerBCG reducing the transmission of $M$. bovis from badgers to cattle by $50 \%$ seems reasonable. Secondly, badgers are responsible for $50 \%$ of bovine TB incidences in cattle in high incidence areas. Of this, initial badger-cattle transmission is $5.7 \%$ and subsequent cattle-cattle transmission is $94.3 \%$ (Donnelly and Nouvellet 2013). Thirdly, 50\% of badgers are vaccinated in high incidence areas of a similar size to the $350 \mathrm{~km}^{2}$ culling areas. Again, this seems reasonable, since the target for the badger culling policy is $70 \%$ of the population. As the AWIA reveals, these inputs for a badger vaccination model lead to a $12.5 \%$ reduction in bovine TB incidence in cattle over 9 years. 
Table 3 Summary AWIA analysis for cattle population

\begin{tabular}{lcccc}
\hline Policy option & \multicolumn{4}{l}{ Impacts (number of cattle) } \\
\cline { 2 - 5 } & \multicolumn{1}{l}{ Life } & Killing/death & Positive welfare & Negative welfare \\
\hline Do nothing & $0^{\mathrm{a}}$ & $92,800^{\mathrm{b}}$ & 0 & $92,800^{\mathrm{c}}$ \\
Badger culling & $17,763^{\mathrm{d}}$ & $75,038^{\mathrm{e}}$ & $17,763^{\mathrm{f}}$ & $75,038^{\mathrm{g}}$ \\
Badger vaccination & $11,600^{\mathrm{h}}$ & $81,200^{\mathrm{i}}$ & $11,600^{\mathrm{j}}$ & $81,200^{\mathrm{k}}$ \\
\hline
\end{tabular}

${ }^{a}$ Largely, AWIA impacts in the table are due to interventions. Hence, the total positive welfare is scored as zero, but it can be assumed that most of the cattle population have a life of net positive value

${ }^{\mathrm{b}}$ Baseline number of cattle slaughtered as bovine TB reactors. 12,800 herd breakdowns (Natural England $2011) \times 7.25$ cattle slaughtered per herd breakdown $=92,800$. The figure of 7.25 cattle slaughtered per herd breakdown is calculated from Defra figures for the total number of cattle slaughtered and the number of herd breakdowns in high incidence areas end June 2014-2016 (Defra and GSS 2016)

${ }^{c}$ All slaughtered cattle experience short-duration, moderate-strong intensity, negative welfare due to transportation, lairage and slaughter process. See e.g. FAWC (2003)

${ }^{\mathrm{d}}$ Number of cattle not slaughtered as breakdowns $=2450$ reduced herd breakdowns $(12,800-10,350=$ 2450) (Natural England 2011, p. 17) $\times 7.25$ (cattle slaughtered per breakdown) $=17,763$

${ }^{\mathrm{e}}$ Figure from Natural England: number of breakdowns (5 years of badger culling based on the Donnelly model $)=10,350($ Natural England 2011, p. 17). 10,350 $\times 7.25$ (cattle slaughtered per breakdown $)=$ 75,038

${ }^{\mathrm{f}}$ Cattle not slaughtered avoid short-duration, moderate-strong intensity, negative welfare due to transportation, lairage and slaughter process

${ }^{\mathrm{g}}$ All slaughtered cattle assumed to experience short-duration, moderate-strong intensity, negative welfare due to transportation, lairage and slaughter process

${ }^{\mathrm{h}}$ Figures based on badger wildlife reservoir causing 50\% cattle reactors (Donnelly and Nouvellet 2013); vaccinating $50 \%$ badger population; assumption $50 \%$ efficacy of reduced badger-cattle M. bovis transmission. 92,800 (cattle slaughtered, do nothing) $\times 50 \%$ (badger wildlife reservoir causing $50 \%$ reactors)

$\times 50 \%$ (vaccinated $50 \%$ badgers) $\times 50 \%$ (assumption of BadgerBCG efficacy) $=11,600$ cattle

${ }^{\mathrm{i}}$ Cattle slaughtered $=$ baseline cattle culled - reduced number of cattle culled due to badger vaccination $=$ $92,800-11,600=81,200$ cattle

${ }^{\mathrm{j}}$ Cattle that would otherwise have experienced short-duration, moderate-strong intensity, negative welfare due to transportation, lairage and slaughter process

${ }^{\mathrm{k}}$ All slaughtered cattle assumed to experience short-duration, moderate-strong intensity, negative welfare due to transportation, lairage and slaughter process

\section{Utilitarian Analysis of Badger Control Policy Options}

\section{An Overview of Utilitarian Theory}

Utilitarianism is a form of consequentialist ethical theory. In consequentialism, right action is dictated by the consequences of an act alone. Utilitarianism was proposed by the British legal reformer Jeremy Bentham. Bentham argued that rather than acting according to a system of arbitrary rules, we should act to maximise pleasure and minimise pain. Hence, Bentham argued that right action is determined by acting according to a single principle, the principle of utility (Bentham 1789/1962). 
Table 4 Summary AWIA analysis for badger population

\begin{tabular}{lcccc}
\hline Policy option & \multicolumn{4}{l}{ Impacts (number of badgers) } \\
\cline { 2 - 5 } & Life & Killing/death & Positive welfare & Negative welfare \\
\hline Do nothing & $0^{\mathrm{a}}$ & $401^{\mathrm{b}}$ & 0 & $40,072^{\mathrm{c}}$ \\
Badger culling & 0 & 85,000 & $401^{\mathrm{d}}$ & $79,275^{\mathrm{e}}$ \\
Badger vaccination & 150 & 0 & $15,027^{\mathrm{f}}$ & $60,715^{\mathrm{g}}$ \\
\hline
\end{tabular}

${ }^{a}$ Largely, AWIA impacts in the table are due to interventions. Hence, the total positive welfare is scored as zero, but it can be assumed that most of the wild badger population have a life of net positive value ${ }^{\mathrm{b}}$ Total badger population in $33 \times 350 \mathrm{~km}^{2}$ areas across 4 years = number of culled badgers (over 4 years)

$\times 100 / \%$ badgers culled $=85,000 \times 100 / 70=121,429$. Number of badgers dying due to bovine $\mathrm{TB}=$ number of $M$. bovis-infected badgers $\times 1 \%=40,072 \times 1 \%=401$ badgers. The assumption is made that $1 \%$ of $M$. bovis-infected badgers with extensive and severe lesions (Jenkins et al. 2008, p. 1350) succumb to the disease

${ }^{\mathrm{c}}$ Number of badgers negatively impacted by $M$. bovis infection: Total badger population $\times 33 \%=$ $121,429 \times 33 \%=40,072$ badgers. Figures based on $33 \%$ of badger population in high incidence areas infected with M. bovis (Donnelly 2013; Jenkins et al. 2008, p. 1358). Infection assumed to cause a medium-duration, mild intensity, negative welfare impact. These badgers are likely to have a life of net positive value because the negative experience of infection is outweighed by other positive value in life. ( $1 \%$ of these $M$. bovis-infected badgers has severe and extensive lesions and suffer, i.e. have a life of net negative value: $40,072 \times 1 \%=401$ badgers.)

${ }^{\mathrm{d}}$ Figure based on $1 \%$ of $M$. bovis-infected badgers suffering from bovine TB. Assuming these badgers have a life of net negative value, i.e. a life not worth living, then culling relieves medium-duration, strong intensity, negative welfare. $40,072 \times 1 \%=401$ badgers

${ }^{\mathrm{e}}$ Figure is sum of negative welfare impacts due to: (a) badgers culled by cage-trapping and shooting experiencing moderate negative welfare due to stress of caging: $85,000 \times 50 \%=42,500$, (b) $7.4-22.8 \%$ of badgers culled by controlled/free-shooting in pilot culls taking longer than 5 min to die after being shot (IEP 2014, p. 50) Central estimate: $85,000 \times 50 \% \times 15.1 \%=6418$, (c) $25 \%$ of badger population experiencing social stress related to perturbation: $121,429 \times 25 \%=30,357$ badgers. Total negative welfare $=42,500+6418+30,357=79,275$. (The figure of 79,275 does not account for negative welfare impacts, both mild and severe, due to $M$. bovis infection in badgers)

${ }^{\mathrm{f}}$ Figure based on $33 \%$ of badgers in high-incidence bovine TB areas infected with $M$. bovis. These are badgers which are infected but still have a life of net positive value. Vaccination prevents $M$. bovis infection or reduces severity (Chambers et al. 2011) and should cause an improvement in welfare. Total population $\times 33 \%$ prevalence $\times 50 \%$ vaccinated population $\times 75 \%$ efficacy BadgerBCG $=121,429 \times$ $33 \% \times 50 \% \times 75 \%=15,027$ badgers. $(1 \%$ of $M$. bovis-infected badgers has severe and extensive lesions and suffer, i.e. have a life of net negative value: $40,072 \times 0.01=401$. Hence, these 401 badgers would substantially benefit from vaccination)

${ }^{\mathrm{g}}$ Number of vaccinated badgers $=$ total population in $33 \times 350 \mathrm{~km}^{2}$ areas $\times 50 \%$ vaccination rate $=$ $121,429 \times 50 \%=60,715$ badgers. Figure based on cage-trapping and vaccinating badgers causing shortduration, moderate intensity, negative welfare. As wild animals, it is assumed badgers experience moderate negative welfare when cage trapped. (The figure of 60,715 does not account for negative welfare impacts, both mild and severe, due to $M$. bovis infection in badgers)

Bentham recognised that his utilitarian philosophy might be applicable not only to humans, but also to sentient animals:

The day may come when the rest of animal creation may acquire those rights which never could have been withholden from them but by the hand of 
tyranny... The question is not, Can they reason? nor Can they talk? but, Can they suffer? (Bentham 1789/1990, p. 136)

J. S. Mill followed Bentham's utilitarian approach, but distinguished between higher and lower pleasures. Mill's utilitarianism aimed at maximising total happiness, rather than pleasure (Mill 1861/1962). Despite Bentham's reference to the capacity to suffer, or sentience, grounding moral status, his work was principally aimed at reform in human society. Singer has applied utilitarianism to the treatment of sentient animals (Singer 1995). Singer argues for radical reforms to humankind's treatment of animals. He claims that our moral status quo is speciesist, since we do not give equality of consideration to nonhuman sentient animals. Singer's utilitarian philosophy ultimately leads him to propose radical reforms, such as the effective abolition of modern livestock agriculture.

Garner has claimed that a theory that grounds moral status not in rationality but in sentiency "is peculiarly suited to incorporate the interests of animals" (Garner 2005 , p. 87). Garner, a rights-based theorist, has described four strengths of utilitarianism. First, the theory has no metaphysical assumptions. Secondly, utilitarianism focuses solely on the consequences of an act. Thirdly, utilitarianism is action-guiding, since the right act is simply that which maximises utility. Fourthly, utilitarianism is a flexible ethical theory, compared to, say, the possibility of rigidity in rights-based theory (Garner 2005, pp. 89, 92).

There is not sufficient space here to discuss in detail the strengths and weaknesses of utilitarian moral theory (see Glover (1990) and Smart and Williams (1973) for further discussion). The utilitarian approach is applied to bovine TB and badger control here for the following reasons. First, utilitarianism is a well-established moral theory. Secondly, as Garner notes, utilitarianism is suited to policy on animals, since it focuses on conscious experience and sentience. Thirdly, policy making is often grounded in approaches informed by utilitarian theory, such as economic cost-benefit analyses. Fourthly, utilitarianism permits sacrificing the interests of one individual for the greater benefit of the whole. In society, animals are generally used for human ends, and their interests are traded with humans and other species. This is clearly the case in bovine TB and badger control policy. Cattle are farmed for human benefit and wildlife can be considered in terms of its utility to human society. The badger culling policy is motivated by the rising economic costs (to humans) of bovine TB in cattle. The following section outlines the utilitarian framework used in this analysis.

\section{Relevant Moral Groups and Value Theory}

Utilitarian theory considers all individuals with relevant interests, i.e. those that are sentient. Hence, the key groups in this analysis are humans, cattle and badgers. Humans can be further subdivided into cattle farmers and public groups. The utilitarian analysis in this paper is conducted mostly with reference to a mental state account of value theory. Impacts on farmers, cattle and badgers are generally 
illustrated in terms of wellbeing and welfare. ${ }^{2}$ Hence, the farmer who experiences a bovine TB breakdown will experience reduced happiness and/or wellbeing due to financial implications and emotional stress. Similarly, a badger that is shot and suffers before it dies, or a group of badgers that experience stress due to social disruption, have a reduced quality of life. For the purpose of illustration, and where it seems more appropriate, the discussion refers to utility in terms of preferences. For instance, the British public would prefer not to cull badgers (Defra 2006). We consider that mental state and preference-/desire-based accounts are closely related. In general, if a moral agent or patient has the preference or desire for something, that thing is of benefit to them, and vice versa. Hence, the farmer prefers to avoid a bovine TB breakdown, and the badger has at least first order desires (DeGrazia 1996) that in general lead to the avoidance of suffering and experience positive welfare states. ${ }^{3}$

\section{The Moral Relevance of Killing Cattle and Badgers}

The analysis here is based on a conventional utilitarian account of the harm of killing. In utilitarian theory, there is no intrinsic value in life. Life permits a sentient being to experience pleasure or pain, or have preferences satisfied or frustrated. Thus, in utilitarian theory, killing is permitted, and even obligatory, if the utility that is lost is replaced by some greater degree of utility (Glover 1990, pp. 119-121). The slaughter of a cow or the culling of a badger with a life of net positive value will result in a loss of utility. All else being equal, the killing of a cow or badger that could be expected to continue with a life of net positive value is, therefore, prima facie morally wrong, simply because it reduces total utility in the world. However, following conventional utilitarian theory, this analysis considers that such value is replaceable. Thus, the killing of badgers could be morally justifiable if it resulted in some greater overall utility, for instance the replacement of this utility by cattle, or an increase in human utility through economic benefits. Hence, in the context of killing for this analysis, utility, in terms of welfare, is interchangeable, and the lives of cattle and badgers are in effect replaceable. ${ }^{4}$

\footnotetext{
${ }^{2}$ Human wellbeing and animal welfare can be conceived as mental state theories in terms of utility for the purposes of this analysis. See e.g. Appleby and Sandøe (2002) and Haynes (2008) for further discussion of value theory in humans and animals.

3 To illustrate that mental state and preference accounts are distinct, consider the following. First, consider a farmer that has a preference for badger culling, when the policy would actually reduce his happiness. This might happen if the farmer was in the periphery of the culling zone, and thus was at risk of perturbation increasing the risk of a breakdown. Secondly, it might also happen if the arguments against culling, e.g. based on the RBCT, turn out to be valid, and badger culling ultimately leads to net losses to farmers, leading to reduced happiness. Thirdly, consider the desire of a badger to access a cattle feed store. In the process of accessing the feed, the badger becomes infected by M. bovis, and ultimately suffers as a result of infection.

${ }^{4}$ Some utilitarians argue against such a straightforward replaceability theory. Singer, a preference utilitarian, claims that since mammals have a degree of self-consciousness, it is a moral harm to kill them and they are not simply replaceable (Singer 1993). Višak, based on prior existence utilitarianism, argues against the replaceability argument and the killing of sentient animals (Višak 2013).
} 


\section{Structure of Utilitarian Analysis}

As the empirical evidence base and the merits of badger control are fiercely contested the analysis has been structured to maximise transparency as far as possible. The first part of the analysis compares badger culling to the do-nothing policy option. Within this, there are three stages. The analysis is first based on the assumption that badger culling achieves a 19\% reduction in bovine TB incidence in cattle in those areas (Natural England 2011). Secondly, the impact of suboptimal culling is considered. ${ }^{5}$ These scenarios are assessed first in the context of a 9-year timeframe, and then by consideration of a longer timeframe. The 9-year timeframe follows the science which the badger culling policy is based on. Consideration of a longer timeframe is necessary for the purposes of a full utilitarian analysis.

The analysis is conducted first over 9 years because this is the timeframe of the current government policy. However, it would be incomplete to omit consideration of a longer timeframe, particularly as there are pertinent moral factors for badger control policy when considering the longer timeframe. In particular, does badger culling need to continue beyond the original 4 years to control bovine TB? To illustrate, it is at least possible that, if 4 years of badger culling was necessary to control and ultimately eradicate bovine TB in cattle (and badgers), it is the right policy in the context of a utilitarian framework. However, at the same time, if it was necessary to cull badgers over a longer timeframe, then a utilitarian analysis might find non-culling approaches superior. ${ }^{6}$

Once the badger culling policy option has been assessed, the analysis moves on to assess the merits of badger vaccination. The stated assumptions for the badger vaccination model are outlined earlier in the AWIA section. As for the badger culling policy option, the analysis considers vaccination in the context of achieving its objectives, suboptimal implementation, and 9-year and longer timeframes.

\section{Determinants of Utility}

In utilitarian ethics, the right policy is that which produces maximum utility. In turn, utility - human wellbeing or animal welfare-is determined by three factors:

1. The population size.

2. The intensity of the wellbeing or welfare.

3. The duration of the wellbeing or welfare. ${ }^{7}$

\footnotetext{
5 There are two reasons to account for the potential impacts of suboptimal culling. Firstly, the perturbation effect indicates that suboptimal culling may increase bovine TB incidence in cattle (Bourne et al. 2007; Woodroffe et al. 2006). Secondly, the pilot culls, and indeed subsequent culls, have failed to meet important targets, for instance culling 70\% of the badger population (IEP 2014).

${ }^{6}$ The Chief Veterinary Officer (CVO) has recommended continued annual culling to maintain badgers at a low population level after four years of culling in the Somerset and Gloucestershire cull areas (Defra 2016b).

${ }^{7}$ In terms of the satisfaction of preferences, utility is similarly determined by first, the size of the population having certain preferences; and secondly, the number and intensity of preferences that are satisfied and frustrated for each moral agent (human)/patient (animal).
} 
At the time of the pilot culls, in England there were approximately 53.5 million humans (ONS 2012), 5.308 million cattle (Defra 2014a) and 220,000 badgers (Natural England 2011). Based on these figures, the ratio of humans to cattle to badger populations is 243:10:1. Furthermore, there are $\sim 7750$ dairy producers in England (AHDB 2016). ${ }^{8}$ Hence, the ratio of humans to cattle to badgers to dairy farmers is 6900:685:28:1. The importance of these relative population sizes is that it is at least possible that the utility of the groups most impacted by badger controlcattle, badgers and farmers - is outweighed by the utility of the much larger human public group. Of course, the groups most affected by policy will have, on an individual basis, more potential for larger changes in utility. Thus, since a farmer has more at stake, he is likely to have far stronger preferences for certain policy options, and his individual wellbeing is set to be impacted more than those of individuals in less affected groups. These points are outlined here merely to illustrate how both relevant population sizes and the intensity or strength of individual preferences or utility changes impact analysis. Utilitarian theory does not prioritise some interests over those of others. Whichever policy option leads to the greatest utility, defined as the sum of human wellbeing and animal welfare, is the right policy on badger control.

\section{Winners and Losers in Badger Control Policy Options}

Based on the AWIA analyses earlier in the paper, Tables 5 and 6 illustrate winners and losers in badger culling and badger vaccination policy options respectively. In the tables, an indicative estimate of change in utility is given by $1+/ 2+/ 3+$ for increasing positive wellbeing/welfare, and $1-/ 2-/ 3-$ for increasing negative wellbeing/welfare. The score ' 0 ' denotes no net change in utility. For all groups listed in the table, there are often subgroups that are variously affected. For instance, successful badger culling policy reduces herd breakdowns for farmers inside the culling area, but may increase incidence immediately outside. Similarly, some members of the public support badger culling, whereas others oppose it. In the case of badger culling in 9-year and longer policy timeframes, the 'public' group has been scored ' $+1 /-1$ ' to illustrate the significant utility gains and losses due to badger culling. The public are (overall) opposed to culling, and so have a net utility loss if culling goes ahead, but gain from reduced public expenditure if that policy successfully reduces bovine TB herd breakdowns. The estimated scores refer to the group under consideration and do not take into consideration its size. The population sizes have been included in Tables 5 and 6 simply for reference and to remind the reader of the significant disparities in group sizes.

\footnotetext{
${ }^{8}$ The figure for dairy farmers is given here for the purposes of illustration. Beef cattle and farmers are also impacted by bovine TB; therefore the figure here for dairy farmers underestimates the total number of cattle farmers.
} 
Table 5 Winners and losers in badger culling

\begin{tabular}{|c|c|c|c|c|c|}
\hline $\begin{array}{l}\text { Policy objective } \\
\text { met? }\end{array}$ & Timeframe & $\begin{array}{l}\text { Public } \\
(53.5 \\
\text { million })\end{array}$ & $\begin{array}{l}\text { Farmers } \\
(7750)\end{array}$ & $\begin{array}{l}\text { Cattle } \\
(5.3 \\
\text { million })\end{array}$ & $\begin{array}{l}\text { Badgers } \\
(220,000)\end{array}$ \\
\hline \multirow{3}{*}{$\begin{array}{l}\text { Effective }(\sim 19 \% \\
\text { reduction bovine } \\
\text { TB })\end{array}$} & Nine-year timeframe ${ }^{a}$ & $1+/ 1-$ & $1+/ 2+$ & $1+$ & $3-$ \\
\hline & $\begin{array}{l}\text { Longer timeframe (continued } \\
\text { culling) }{ }^{\mathrm{b}}\end{array}$ & $1+/ 1-$ & $1+$ & $1+$ & $3-$ \\
\hline & $\begin{array}{l}\text { Longer timeframe (no further } \\
\text { culling) }{ }^{\mathrm{c}}\end{array}$ & $1+$ & $2+$ & $1+$ & $0 /-1$ \\
\hline \multirow{2}{*}{$\begin{array}{l}\text { Ineffective (neutral/ } \\
\text { minimal positive } \\
\text { effect on bTB) }\end{array}$} & Nine-year timeframe ${ }^{\mathrm{d}}$ & -1 & -1 & 0 & $3-$ \\
\hline & Longer timeframe $\mathrm{e}^{\mathrm{e}}$ & -1 & -1 & 0 & $3-$ \\
\hline \multirow{2}{*}{$\begin{array}{l}\text { Deleterious effect } \\
\text { (increase/worsen } \\
\text { bTB) }\end{array}$} & Nine-year timeframe ${ }^{\mathrm{f}}$ & $2-$ & $2-$ & $2-$ & $3-$ \\
\hline & Longer timeframe $^{\mathrm{g}}$ & $2-$ & $2-$ & $2-$ & $3-$ \\
\hline
\end{tabular}

aPublic set to gain from reduced expenditure, but majority opposed to culling. Cattle farmers' utility gain of reduced bovine TB incidence tempered by economic costs of policy and public opposition related issues potentially affecting beef and dairy consumption. Cattle benefit from reduced culling rates. Badgers lose due to culling and substantial reduction in population size

${ }^{\mathrm{b}}$ The longer timeframe and continued culling mirrors the 9-year timeframe, aside from potential reduced utility to farmers arising from increasing public opposition to ongoing and indefinite cull

${ }^{c}$ Public overall positive utility because earlier opposition to 4-year cull outweighed by tax/public expenditure savings in longer term. Badger utility would be related to whether prior pre-cull population levels are re-established and whether longer term positive benefits of being $M$. bovis-free outweigh negative utility associated with culling and reduced population

${ }^{\mathrm{d}}$ Ineffective badger culling has large negative utility impacts for badgers. Farmers are negatively impacted due to badger culling and no associated benefit, with likely negative consumer response and therefore economic impact

${ }^{\mathrm{e}}$ Longer ineffectual timeframe mirrors 9-year ineffectual timeframe

${ }^{\mathrm{f}}$ Deleterious effect of badger culling leads to moderate size negative utility impacts for all parties, except the badger population which has a large magnitude change, mostly based on population size changes as in above scenarios

${ }^{\mathrm{g}}$ Longer timeframe for deleterious culling mirrors 9-year timeframe

\section{Utility in the Do-Nothing and Badger Culling Policy Options}

\section{Key Figures: Policy Impacts, Economics and Public Opinion}

In the do-nothing policy option, the number of cattle slaughtered due to bovine TB over 9 years is 92,800. In badger culling, Natural England has estimated around 85,000 (70,000-100,000) badgers will be culled over 4 years (Natural England 2011 , p. 5). Based on data from the RBCT, around 33\% of badgers in high incidence areas are infected with $M$. bovis, and $1 \%$ of these have extensive and severe TB lesions (Jenkins et al. 2008). The $33 \%$ of badgers mildly infected with bovine TB are considered to have a reduced quality of life due to infection, but still have a life of net positive value. The $1 \%$ of badgers that have extensive and severe TB lesions are considered to have a life of net negative value, i.e. a life not worth living (FAWC 2009a). 
Table 6 Winners and losers in badger vaccination

\begin{tabular}{llllll}
\hline Policy objective met? & Timeframe & $\begin{array}{l}\text { Public (53.5 } \\
\text { million })\end{array}$ & $\begin{array}{l}\text { Farmers } \\
(7750)\end{array}$ & $\begin{array}{l}\text { Cattle (5.3 } \\
\text { million) }\end{array}$ & $\begin{array}{l}\text { Badgers } \\
(220,000)\end{array}$ \\
\hline $\begin{array}{l}\text { Effective }(\sim 12.5 \% \text { reduction } \\
\text { bTB) }\end{array}$ & $\begin{array}{c}\text { Nine-year } \\
\text { timeframe } \\
\text { Longer } \\
\text { timeframe }\end{array}$ & $1+$ & $2+$ & $1+$ & $1+$ \\
$\begin{array}{c}\text { Ineffective (neutral or minimal } \\
\text { positive effect on bTB) }\end{array}$ & $\begin{array}{c}\text { Nine-year } \\
\text { timeframe }\end{array}$ \\
$\begin{array}{c}\text { Longer } \\
\text { timeframe }\end{array}$ & 0 & 0 & $1+$ & 0 & 0 \\
\hline
\end{tabular}

${ }^{a}$ Effective badger vaccination mild-moderate positive utility changes across all groups: public due to tax/ public expenditure savings; cattle farmers due to economic savings not tempered by reputational damage; cattle and badgers both have increases in utility associated with reduced disease and its consequences

${ }^{\mathrm{b}}$ Longer timeframe for badger vaccination mirrors 9-year timeframe

${ }^{c}$ Ineffectual badger vaccination has insignificant utility impacts across all groups

${ }^{\mathrm{d}}$ Longer timeframe ineffectual badger vaccination mirrors 9-year policy timeframe

From 2004 to 2014, bovine TB control has cost the government, and therefore the taxpaying public, around $£ 500$ million, and the figure is set to double over the next decade. It has been estimated that each TB breakdown costs government $£ 20,000$ and the farmer $£ 10,000$ (Defra 2011a, p. 16). Herd breakdowns cause farmers considerable financial cost and emotional stress (FCN 2009).

In a Defra 2006 public consultation on badger culling, 95.6\% $(45,415 / 47,308)$ of respondents were opposed to culling. A 2010 government consultation revealed the following: $61 \%$ for badger vaccination and against badger culling; $30 \%$ for badger vaccination and badger culling; $8 \%$ against badger vaccination and against badger culling (Defra 2011b, p. 2). In 2013 over 300,000 people signed a government e-petition to stop the badger cull (HM Gov 2013). A parliamentary backbench motion against the cull which followed the petition was passed by 147 to 28 votes (HC Deb 2013). A backbench motion on 14 March 2014 against the cull, after the findings of the IEP report were leaked, passed by 219 votes to 1 (HC Deb 2014).

\section{Cattle and Badger Utility}

Cattle and badger utility is influenced by population- and welfare-based considerations. In badger culling, given a $19 \%$ reduction in bovine $\mathrm{TB}$, badger culling results in 17,763 fewer cattle slaughtered over 9 years. Utilitarian theory considers life to have instrumental and not intrinsic value. Hence, the utility of sentient animals is considered to be replaceable. Most (perhaps all) of these culled cattle will be replaced by other cattle, so there will be at most only small changes in total cow utility, which arise from welfare considerations-principally due to an increased rate of culling as well as more tuberculin testing.

However, the UK dairy herd has been in decline in recent decades. There are no data on the contribution of bovine TB to this long-term trend, but it can be assumed 
that it is to some extent a factor. Provided cattle have a life of net positive value, reduced national herd size contributes to a decrease in utility.

The slaughter of 17,763 fewer cattle means the process of transport, lairage and slaughter, which causes short-duration, medium-strong intensity, negative welfare, is avoided for this number of cattle over 9 years (FAWC 2003). Hence, reduced cattle culling rates results in increased utility due to avoiding stress associated with slaughter. Testing is likely to cause short-duration, mild intensity, negative welfare for cattle. Based on the long-term policy goal of eradication, we assume there will be no significant changes to national and regional compulsory testing intervals of cattle over 9 years. Hence, testing intervals are not relaxed in high incidence areas during the 9-year policy timeframe. However, a reduction in bovine TB incidence will ultimately result in reduced testing on farms due to fewer reactors. ${ }^{9}$

Natural England has estimated that badger culling will reduce the badger population by up to $30 \%$ across the whole of England and up to $50 \%$ in the west and south-west regions (Natural England 2011, p. 2). Hence, badger culling may cause a net 66,000 (from 220,000 to 154,000) reduction in England's badger population. In this analysis, it is assumed that the $1 \%$ (401) of badgers with extensive and severe lesions experience net negative welfare, i.e. they do not have a life worth living (FAWC 2009a). Research suggests the majority of badgers infected with M. bovis do not suffer, based on weight gain, life span and ability to reproduce (Defra 2010a). ${ }^{10}$

The IEP has reported that 7.4-22.8\% (central estimate $15.1 \%$ ) of badgers in the pilot culls took over $5 \mathrm{~min}$ to die (IEP 2014, p. 50). Based on these figures, 6418 badgers experience short-duration, strong intensity, negative welfare due to suboptimal culling by controlled/free-shooting. Given the assumption of $50 \%$ of badgers being culled by cage-trapping and shooting, 42,500 badgers experience short-duration, moderate intensity, negative welfare due to cage trapping. The perturbation effect arises because "disruption of badger's territorial system" causes wider ranging behaviour of badgers (Bourne et al. 2007, p. 85). It is likely that the disruption caused by badger culling is associated with stress to the local badger population. The AWIA estimates that $25 \%(30,357)$ of the culled badger population experience medium-duration, moderate intensity, negative welfare impact associated with culling. ${ }^{11}$

All else being equal, the $50 \%$ reduction in badger population in the west and south-west regions causes a $50 \%$ reduction in badger utility. The negative utility due to direct welfare consequences of culling (12,835 badgers) and stress associated with perturbation (30,357 badgers) will further reduce badger utility. One percent,

\footnotetext{
9 A 'reactor' is a cow which reacts to an injection of tuberculin antigen, i.e. tests positive for M. bovis infection/exposure.

${ }^{10}$ E.g. Defra states the following in an annex to its 2010 public consultation on badger control: "Badgers infected with TB rarely suffer from the infection. Infected badgers are able to reproduce and raise young successfully and live for several years. The lifespan of a badger is relatively short (average 3-5 years), and so very few badgers will reach the terminal clinical stages of disease" (Defra 2010a, p. 3).

11 Given that the target is to cull $70 \%$ of the badger population in culling areas, the figure of $25 \%$ is very conservative, and it is likely that a significantly higher proportion of the population may experience negative welfare due to social disruption as a result of culling over the period of the cull.
} 
i.e. 401 badgers with a life of net negative welfare, will benefit by being culled, since their death relieves a life of suffering.

Badger culling therefore causes an ongoing loss of utility due to the reduction in the badger population (by 66,000), and additional negative utility impacts for badgers (direct and indirect consequences of culling). For cattle, it reduces shortduration, moderate-strong intensity, negative utility due to the slaughter process $(17,763$ cattle). Based on a stable cattle population, it is clear from these figures that badger culling would result in the net negative utility change of the badger population outweighing the net positive utility change of the cattle population. If bovine TB contributes to the declining cattle population, the comparison of utility for cattle and badgers will ultimately depend on how much of the decline in cattle population is related to it. The authors are not aware of any data on this. Bovine TB is likely to have some influence. However, the long-running low farm-gate price of milk probably has a greater impact.

\section{Human Utility}

If badger culling were to achieve a $19 \%$ reduction in bovine TB in cattle, the utility of farmers negatively impacted by the disease would be increased. This increase in utility would partly be due to the positive feeling resulting from improving disease conditions and a sense of control over their future in being able to control the wildlife reservoir of infection. In terms of preferences, utility increases simply due to the satisfaction of farmers' preferences for a badger culling policy, together with associated preferences, such as the preference to avoid a TB breakdown.

A key driver of badger culling is cost savings for farmers as a consequence of fewer breakdowns. The Defra 2010 impact assessment, which preceded the start of the badger cull, estimated that a farmer- and landowner-led cull would save $£ 0.57$ million per $150 \mathrm{~km}^{2}$ for farmers (Defra 2010b, p. 8). However, Defra's economic costing of badger culling has been challenged. For instance, costs to police the 2013 pilot cull were $£ 1311$ per badger, double the original estimates (BBC News 2014). The IEP reported that cage-trapping to cull was used extensively in the pilot culls (IEP 2014, p. 6). Cage-trapping is over eight times more costly than free-shooting (Defra 2011d, p. 6). Jenkins et al. have claimed that "on the basis of costeffectiveness" it is unlikely badger culling can contribute to disease control (Jenkins et al. 2010, p. 6). Indeed, prior to the pilot culls, Defra may have foreseen the problem of economic justification for badger culling, at least in the short term:

Even if the experience of culling in the pilot areas provided evidence that culling could only be carried out at a net cost to Government and the farming industry, this would not necessarily undermine the case for wider roll-out for the purposes of preventing the future spread of disease. (Defra 2011c, pp. $16-17)^{12}$

\footnotetext{
${ }^{12}$ Defra's more recent cost benefit analysis estimates the total quantified benefits at $£ 2.59 \mathrm{~m}$ and the total cost $£ 2.03 \mathrm{~m}$. Hence, the department estimates the economic benefits to outweigh the costs by $£ 0.55 \mathrm{~m}$ per culling area, but advises there is considerable uncertainty about the figures (Defra 2016a, p. 7).
} 
Natural England has estimated that badger culling, given a $19 \%$ reduction in bovine TB incidence, will result in an overall reduction of 2450 herd breakdowns over 9 years. The agency estimates that an individual farmer would have a 1 in 5 chance of avoiding a breakdown he would otherwise experience. If the farm was within the culling area there would be a 3 in 10 chance of avoiding the breakdown he would otherwise experience (Natural England 2011, p. 17).

Impacts on farmers are uncertain due to the question of whether there would in reality be a significant net financial benefit. As discussed above, calculations have shown that the costs of the policy may be greater than the benefits. ${ }^{13}$ Furthermore, there is considerable uncertainty about whether implementation of the policy can be successful. There is also a question of the wider consequences of the public's perception of an ongoing cull and the impact this may have on the purchasing behaviour of beef and dairy products. For instance, after the government announced a badger culling policy, supermarkets were asked to make statements on milk supply policy with respect to culls (Smithers 2012). Animal protection NGO and consumer pressure on supermarkets is likely to continue and may intensify when culls are rolled out.

Government consultations, public polls and parliamentary debates strongly suggest that a large majority of the public are opposed to a badger cull. The utility of the public opposed to a cull is likely to be decreased in the event of a cull and increased in the event of a no-cull policy. Of course, many of those opposed to a cull are likely to have only a small decrease in overall utility if the cull goes ahead. However, some of those opposed to culling, as a result of the strength of their convictions, are likely to have a more substantial decrease in utility.

Concentrating on human utility alone, is the gain in human utility of those set to benefit from a cull greater than the loss? This is a complex empirical question, with a significant degree of uncertainty. The question can only be decided upon by making clear the impacts of badger culling on all affected groups. Restricting this part of the analysis to human utility alone, the judgement here is that total utility will be greater in the event of a non badger-culling policy.

A key reason for this judgement relates to the scientific uncertainty and the potential economic benefits of the cull. The ISG, the group which oversaw the 8-year RBCT, recommended against badger culling. Arguably, the ISG's key recommendations relating to bovine $\mathrm{TB}$ and badger culling have been borne out since it reported almost a decade ago. First, the ISG reported the difficulties of culling a large proportion of badgers over a very short timeframe. The 2013 pilot culls both fell well short of the $70 \%$ cull targets, during the 6 week cull licence (IEP 2014). ${ }^{14}$ Secondly, the ISG warned that other culling methods, such as controlled/ free-shooting, could not be relied on to be effective. The IEP reported that a large proportion of culling in 2013 was conducted by cage-trapping, despite this method being significantly more expensive than controlled/free-shooting methods. Thirdly,

\footnotetext{
13 The costs to implement the cull are met by the farming industry. Government covers surveillance and policing costs.

14 The significant extensions to culling periods granted in both areas are likely to have resulted in an increased level of badger perturbation with an associated reduction in the benefits of culling in terms of reducing the incidence of bovine $\mathrm{TB}$ in cattle.
} 
the ISG reported that bovine TB could be brought under control, and ultimately eradicated, by cattle-based measures alone. In Wales, the government made cattlebased measures significantly more stringent from 2008. Subsequently, from 2008-2015, the bovine TB incidence in cattle has fallen by some 50\%, a far greater figure than the $19 \%$ projection based on badger culling in high incidence areas in England (APHA 2015, pp. 6, 18).

\section{Badger Culling and Utility Beyond 9 Years}

Assumptions and uncertainty will increase with longer timeframes and predictions will become less precise. If badger culling becomes a long-term policy, much of the above analysis is likely to hold in broad terms, even if some of the parameters change. It has been British policy to cull badgers for a large part of the last 40 years. There has been considerable public opposition to culling for much of this time. Therefore, it is reasonable to assume that a majority of the public will remain opposed to badger culling, and a significant proportion will have strong convictions about the issue. The longer-term utility calculations will also depend on the feasibility of elimination of bovine TB in England. For instance, consider if badger culling is necessary to control bovine TB incidence in the medium term (e.g. over 9 years), but that after culling for several years, there is no need for further culling, and the disease in cattle and badgers is brought under control. In such a scenario, the improvement in utility of future generations of badgers will at some point outweigh the negative utility caused to the current and next generations of badgers through badger culling. ${ }^{15}$ In this way, the welfare of current generations of badgers in high risk areas is in effect sacrificed for cattle and future generations of badgers. However, it is also important to remember that any longer-term increases in individual badger utility (due to reduced $M$. bovis infection prevalence) at any given point in time would, at least in the medium term, be accompanied by a significantly reduced badger population, which would have the effect of reducing total badger utility. $^{16}$

Ultimately, the long-term feasibility of eradication of bovine TB may depend on developments in technology, particularly a cattle vaccine and a validated DIVA (differentiate infected from vaccinated animals) test. Additionally, political factors, such as EU acceptance of cattle vaccination, will play an important role ${ }^{17}$ (Defra 2014b).

\footnotetext{
15 It is this sort of scenario that leads to claims made by pro-culling actors that badger culling is actually good for badgers. However, it is important to state that the science does not appear to support such a scenario. Repeated badger culling has been shown to increase the prevalence of bovine TB in badger populations (Woodroffe et al. 2006).

16 This is a good example of the distinction between total and average utilitarianism. In this scenario it is plausible that a smaller number of badgers will have a greater average utility, but the utility of the total population is less, since the badger population size has been reduced.

17 In 2016, the UK public voted to leave the EU and at the time of writing (July 2017) it is the stated policy of the Westminster government to implement Brexit. However, should the UK leave the EU, it will almost certainly continue with a bovine TB eradication policy, not least because the EU will continue to be a major trading partner.
} 


\section{Utility in Vaccination of Badgers}

The utility of badger vaccination will depend on two factors: first, the impact of BadgerBCG on transmission of $M$. bovis from badgers to cattle; and secondly, the feasibility of vaccinating a large number of badgers on an annual basis. ${ }^{18}$ The AWIA model has assumed that vaccination of badgers reduces the transmission rate of $M$. bovis from badgers to cattle by $50 \%$. It also assumes that badgers cause $50 \%$ of herd breakdowns in high risk areas and that $50 \%$ of the badger populations are vaccinated. Based on these figures, the AWIA finds that badger vaccination can achieve a reduction of 11,600 cattle slaughtered. This equates to 1,600 fewer herd breakdowns, based on 7.25 cattle culled per breakdown (Defra and GSS 2016), which represents a $12.5 \%$ decrease in bovine TB incidence. This compares to 17,763 fewer cattle slaughtered as a result of 2450 fewer breakdowns if the $19 \%$ reduction in bovine TB incidence is achieved by badger culling.

If badger vaccination is nearly as effective as this, then it is the optimal policy option from a utilitarian perspective. Badger vaccination does not cause a substantial reduction in the badger population, whereas the badger culling policy option does. The various risk factors reducing human utility in the badger culling option do not apply to badger vaccination. In particular, badger vaccination is supported by majority public opinion (Defra 2011b, p. 2). As badger vaccination is supported by public opinion, there are no risks to the farming industry associated, for example, with supermarkets sourcing milk from non-badger cull areas or segments of the public reducing beef and dairy consumption.

Furthermore, despite uncertainty about the efficacy of badger vaccination, there is minimal risk associated with perturbation (Bourne et al. 2007, p. 152). Badger culling can increase badger-cattle transmission of $M$. bovis by perturbation, and increase the prevalence of $M$. bovis-infected badgers in the surviving population (Woodroffe et al. 2006, pp. 1, 2, 8). In contrast, vaccinating badgers does not remove them from the population; therefore the negative effects of perturbation do not arise (Bourne et al. 2007, p. 152).

Defra found the costs of cage-trapping to vaccinate to be higher than freeshooting ( $£ 2250 / \mathrm{km}^{2} /$ year vs. $£ 300 / \mathrm{km}^{2} /$ year) (Defra 2011d, p. 6). Despite this, the free-shooting method used in the 2013 pilot culls failed to kill a sufficient number of badgers and the pilot culls resorted to cage-trapping and shooting. It has been reported that only $24 \%$ of badgers were killed using free-shooting (Press Association 2014). The cost of cage-trapping to vaccinate is slightly less than cage-trapping to cull ( $£ 2250 / \mathrm{km}^{2} /$ year vs. $£ 2500 / \mathrm{km}^{2} /$ year).

There is limited scientific evidence about the relative impact of badger culling versus vaccination on M. bovis transmission. The ISG states in its Final report:

If vaccination could reduce $M$. bovis transmission among badgers, and from badgers to cattle, this might have an overall beneficial effect on cattle herd breakdowns greater than that achieved by culling. (Bourne et al. 2007, p. 152)

\footnotetext{
${ }_{18}$ The BadgerBCG vaccine must be administered by cage trapping and vaccinating annually. In badger culling, the objective is to cull $70 \%$ of the badger population in the first year of culling, and then maintain the population below this level in each of three further years of culling.
} 
Chambers et al. have found that BCG vaccination reduces the sero-prevalence of $M$. bovis in badgers by $73.8 \%$ (Chambers et al. 2011, p. 1913). Defra has stated that there is no evidence that vaccination of badgers protects badgers already infected with M. bovis (Defra 2011c, p. 7). This analysis assumes 33\% of badgers in high incidence areas are infected with $M$. bovis (Jenkins et al. 2008, p. 1530). Hence, BadgerBCG is likely to have some efficacy in up to $67 \%$ of badgers in high incidence areas. Badgers have an average lifespan of 3-5 years and 30\% of the population is turned over annually. Therefore, $30 \%$ of infected badgers die annually and those badgers infected at the beginning of the policy timeframe will have died out within 3-5 years (Godwin-Pearson 2012, p. 19).

Finally, the phenomenon of herd immunity means that not all badgers will need to be protected for vaccination (Defra 2011c, p. 7). In terms of comparison of policy options, badger vaccination needs only to achieve a $19 \%$ reduction in cattle reactors to be superior to badger culling based on the simple objective of reducing TB in cattle. There is nothing from an epidemiological perspective that makes this outcome particularly unrealistic. When the utility costs of badger culling are factored in-reduced utility of badgers and the public opposed to the cull-the claim that vaccination is the optimal policy option is reasonably well-grounded.

\section{Conclusion}

Bovine TB is an important animal health issue in Britain. In England, the disease is particularly controversial due to the Westminster government's badger culling policy. The scientific justification to cull badgers is highly contested. The ISG, based on the government-commissioned RBCT, recommended against badger culling. In contrast, the King review found that badger culling could contribute to controlling bovine TB in cattle. The policy issue is also highly controversial because the badger is a cherished part of wildlife with an important place in British culture.

This paper has argued that bovine TB and badger control policy should not, and indeed cannot, be made simply based on the scientific evidence base, or by use of economic cost benefit analysis. Ultimately, policy on badger control is necessarily a moral issue and should be addressed in the context of the following question: Ethically, what is the right, or most justifiable, policy on badger control considering impacts on all morally relevant affected groups? An informed answer to this question requires, first, Animal Welfare Impact Assessment (AWIA) and, secondly, independent expert ethical analysis. The AWIA is a policy tool which estimates the life and death, and positive and negative welfare impacts on each species of animal impacted by all policy options. Independent ethical analysis should be conducted in established frameworks by a committee with expertise in ethics.

In this paper, a summary AWIA has been provided of bovine TB and badger control policy options of (1) do nothing, (2) badger culling, and (3) badger vaccination. We have applied a utilitarian ethical framework to these policy options. The paper finds that non-culling approaches, including the do-nothing and badger vaccination policy options, are superior to the badger culling option. A badger vaccination model that results in a $12.5 \%$ reduction in the incidence of bovine TB 
over 9 years will result in greater utility, in terms of human wellbeing and animal welfare, than a badger culling programme that results in a $19 \%$ reduction over the same time period. Badger culling results in a $30 \%$ reduction in the size of the badger population in England, as well as causing direct and indirect welfare impacts due to the culling process. Additionally, there is significant public opposition to badger culling. All of these factors will reduce total utility, even if badger culling achieves its objective to reduce bovine TB incidence by $19 \%$ over 9 years. Finally, there are considerable empirical uncertainties associated with badger culling. These include suboptimal culling causing perturbation, and thus worsening the disease in both species, economic costs of culling, and consumer purchasing behaviour related to opposition to culling.

Acknowledgements The first author would like to thank the Royal Veterinary College, which funded work for a Ph.D. project that this paper is based on. He would also like to thank animal health and welfare policy actors interviewed during the course of the research. We would like to thank the late Christopher Wathes, who supervised the earlier part of the first author's Ph.D. research, which this paper is based on.

Open Access This article is distributed under the terms of the Creative Commons Attribution 4.0 International License (http://creativecommons.org/licenses/by/4.0/), which permits unrestricted use, distribution, and reproduction in any medium, provided you give appropriate credit to the original author(s) and the source, provide a link to the Creative Commons license, and indicate if changes were made.

\section{References}

AHDB. (2016). UK producer numbers. https://dairy.ahdb.org.uk/market-information/farming-data/ producer-numbers/uk-producer-numbers/.

APHA. (2015). Epidemiology of bovine tuberculosis in Wales: Annual surveillance report. London: Animal and Plant Health Agency.

Appleby, M. C., \& Sandøe, P. (2002). Philosophical debate on the nature of well-being: Implications for animal welfare. Animal Welfare, 11(3), 283-294.

Battersby, J., \& Partnership, Tracking Mammals. (2005). UK mammals: Species status and population trends. JNCC/Tracking Mammals Partnership: First report by the Tracking Mammals Partnership. Peterborough.

BBC News. (2014). Policing badger cull cost ' $£ 1,311$ per badger'. BBC http://www.bbc.co.uk/news/ukengland-25719562.

Bentham, J. (1789/1962). An introduction to the principles of morals and legislation. In M. Warnock (Ed.), Utilitarianism (pp. 33-77). The University of California: Fontana.

Bentham, J. (1789/1990). An introduction to the principles of morals and legislation. In A. Lindzey \& P. B. Clarke (Eds.), Animal rights: A historical anthology (pp. 135-137). New York: Columbia University Press.

Bourne, F. J., Donnelly, C. A., Cox, D. R., Gettinby, G., McInerney, J. P., Morrison, W. I., et al. (2007). Bovine TB: The scientific evidence-final report of the independent scientific group on cattle TB. London: Independent Scientific Group on Cattle TB.

British Wildlife Centre. (2012). British wildlife species collection: Badger —Meles meles. Retrieved 1 February, 2015, from http://www.britishwildlifecentre.co.uk/planyourvisit/animals/badger.html.

Chambers, M. A., Rogers, F., Delahay, R. J., Lesellier, S., Ashford, R., Dalley, D., et al. (2011). Bacillus Calmette-Guérin vaccination reduces the severity and progression of tuberculosis in badgers. Proceedings of the Royal Society B: Biological Sciences, 278(1713), 1913-1920.

DairyCo. (2014a). UK cow numbers. Retrieved 2 February, 2015, from http://www.dairyco.org.uk/ market-information/farming-data/cow-numbers/uk-cow-numbers/. 
DairyCo. (2014b). World dairy cow numbers. Jan 2014. Retrieved 2 April, 2014, from http://www. dairyco.org.uk/market-information/farming-data/cow-numbers/world-cow-numbers/.

Defra. (2006). Public consultation on controlling the spread of bovine tuberculosis in cattle in high incidence areas in England: Badger culling-Summary of responses. London: Defra.

Defra. (2010a). Bovine tuberculosis: The government's approach to tackling the disease and consultation on a badger control policy-Annex $G, Q \& A$. London: Defra.

Defra. (2010b). Measures to address Bovine TB in baders: Impact assessment, consultation stage. London: Defra.

Defra. (2011a). Bovine TB eradication programme for England. London: Defra.

Defra. (2011b). Bovine tuberculosis: The government's approach to tackling the disease and consultation on a badger control policy-Summary of consultation responses. London: Defra.

Defra. (2011c). The government's policy on bovine TB and badger control in England. London: Defra.

Defra. (2011d). Measures to address bovine TB in badgers: Impact assessment. London: Defra.

Defra. (2013). Farming statistics-Livestock populations at 1st December 2012, UK and England. London: Defra Retrieved from https://www.gov.uk/government/uploads/system/uploads/ attachment_data/file/183227/defra-stats-foodfarm-landuselivestock-farmstats-dec2012-130314.pdf.

Defra. (2014a). Farming statistics—Livestock populations at 1 December 2013, England. London: Defra Retrieved from https:/www.gov.uk/government/uploads/system/uploads/attachment_data/file/ 286876/structure-dec2013-eng-06mar14.pdf.

Defra. (2014b). The strategy for achieving officially bovine tuberculosis free status for England. London: Defra.

Defra. (2016a). Badger control policy: Value for money analysis 2016. London: Defra.

Defra. (2016b). Chief veterinary officer's advice on the outcome of the 2016 badger culls. London: Defra.

Defra. (2016c). Further measures to eradicate bovine TB [press release]. Retrieved from https://www.gov. uk/government/news/further-measures-to-eradicate-bovine-tb.

Defra. (2016d). Summary of badger control monitoring during 2016. London: Defra.

Defra, \& GSS. (2016). Quarterly publication of National Statistics on the incidence and prevalence of tuberculosis (TB) in Cattle in Great Britain-To end June 2016. London: Office for National Statistics.

DeGrazia, D. (1996). Taking animals seriously: Mental life and moral status. Cambridge: Cambridge University Press.

Donnelly, C. (2013). The badger cull-Key science questions answered. The guardian. Retrieved from http://www.theguardian.com/environment/2013/oct/11/badger-cull-key-science-questions-answered.

Donnelly, C. A., \& Nouvellet, P. (2013). The contribution of badgers to confirmed tuberculosis in cattle in high-incidence areas in England. PLOS Currents Outbreaks. doi:10.1371/currents.outbreaks. 097a904d3f3619db2fe78d24bc776098

FAO. (2010). Statistical yearbook of the Food and Agricultural Organization: Food and Agricultural Organization.

FAWC. (2003). Report on the welfare of farmed animals at slaughter or killing. London: Farm Animal Welfare Council.

FAWC. (2009a). Farm animal welfare in Great Britain: Past, present and future. London: Farm Animal Welfare Council.

FAWC. (2009b). Opinion on the welfare of the dairy cow. London: Farm Animal Welfare Council.

FCN. (2009). Stress and Loss: A report on the impact of bovine TB on farming families. Northampton: Farm Crisis Network.

Garner, R. (2005). Animal ethics. Cambridge: Polity Press.

Glover, J. (Ed.). (1990). Utilitarianism and its critics. New York: Macmillan.

Godwin-Pearson, G. (2012). Common sense and bovine TB: Why the government should abandon badger culling trials in favour of vaccination. London: The Bow Group.

Haynes, R. P. (2008). Animal welfare: Competing conceptions and their ethical implications. New York: Springer.

HC Deb. (2013). Badger Cull. Hansard, 25 Oct 2013: Column 1095.

HC Deb. (2014). Badger Cull. Hansard, 577(col. 456).

HM Gov. (2013). Stop the Badger Cull: e-petition. Retrieved 2 Jan 2017 http://epetitions.direct.gov.uk/ petitions/38257.

IEP. (2014). Pilot badger culls in Somerset and Gloucestershire: Report by the Independent Expert Panel. London: Independent Expert Panel. 
Jenkins, H. E., Morrison, W. I., Cox, D. R., Donnelly, C. A., Johnston, W. T., Bourne, F. J., et al. (2008). The prevalence, distribution and severity of detectable pathological lesions in badgers naturally infected with Mycobacterium bovis. Epidemiology and Infection, 136(10), 1350-1361.

Jenkins, H. E., Woodroffe, R., \& Donnelly, C. A. (2010). The duration of the effects of repeated widespread badger culling on cattle tuberculosis following the cessation of culling. PLoS ONE, 5(2), e9090. doi:10.1371/journal.pone.0009090.

King, D. (2007). Bovine tuberculosis in cattle and badgers. London: Defra.

McCulloch, S. P. (2015). The British animal health and welfare policy process: Accounting for the interests of sentient species. (Ph.D.), University of London.

McCulloch, S. P., \& Reiss, M. J. (2017). Bovine tuberculosis and badger control in Britain: Science, policy and politics. Journal of Agricultural \& Environmental Ethics. doi:10.1007/s10806-017-96863.

Messenger, S. (2016). 'Refreshed' bovine TB policy includes targeting infected badgers. BBC News,. Retrieved from http://www.bbc.co.uk/news/uk-wales-37679272.

Mill, J. S. (1861/1962). Utilitarianism. In M. Warnock (Ed.), Utilitarianism (pp. 251-321). London: Fontana.

Natural England. (2011). The impact of culling on badger (Meles meles) populations in England and measures to prevent their 'local disappearance' from culled areas: Supplementary advice provided under the Protection of Badgers Act 1992 and Wildlife \& Countryside Act 1981 (as amended): Natural England.

ONS. (2012). Total Population (UK). Retrieved 6 March, 2014, from http://www.ons.gov.uk/ons/ taxonomy/index.html?nscl=Population\#tab-overview.

Press Association. (2014). Badger cull killed only 24\% of animals by controlled shooting, figures show. The guardian. Retrieved from http://www.theguardian.com/environment/2014/jan/23/badger-cullonly-quarter-controlled-shooting.

Singer, P. (1993). Practical ethics (2nd ed.). Cambridge: Cambridge University Press.

Singer, P. (1995). Animal liberation. London: Pimlico.

Smart, J. J. C., \& Williams, B. (1973). Utilitarianism: For and against. Cambridge: Cambridge University Press.

Smithers, R. (2012). Supermarkets face protests over support for badger cull. The guardian. Retrieved from http://www.theguardian.com/environment/2012/sep/25/supermarkets-protests-badger-cull.

Višak, T. (2013). Killing happy animals: Explorations in utilitarian ethics. London: Palgrave Macmillan.

Wang, A. (2011). Meles meles: Eurasian badger. Available from University of Michigan Animal Diversity Web Retrieved 5 February 2015 http://animaldiversity.ummz.umich.edu/accounts/Meles_ meles/\#lifespan_longevity.

Welsh Government. (2017). Strengthened approach to tackling bovine TB in Wales announced. Retrieved 30 June, 2017, from http://gov.wales/newsroom/environmentandcountryside/2017/170620strengthened-approach-to-tackling-bovine-tb-in-wales-announced/?lang=en.

WildCRU. (2015). Research: The badger project. Retrieved 5 February 2015, 2015, from http://www. wildcru.org/research/the-badger-project/.

Wilson, G., Harris, S., \& McLaren, G. (1997). Changes in the British badger population, 1988-1997. London: People's Trust for Endangered Species.

Woodroffe, R., Donnelly, C. A., Cox, D. R., Bourne, F. J., Cheeseman, C. L., Delahay, R. J., et al. (2006a). Effects of culling on badger Meles meles spatial organization: Implications for the control of bovine tuberculosis. Journal of Applied Ecology, 43(1), 1-10. doi:10.1111/j.1365-2664.2005. 01144.x.

Woodroffe, R., Donnelly, C. A., Jenkins, H. E., Johnston, W. T., Cox, D. R., Bourne, F. J., et al. (2006b). Culling and cattle controls influence tuberculosis risk for badgers. Proceedings of the National Academy of Sciences of the United States of America, 103(40), 14713-14717. doi:10.1073/pnas. 0606251103 . 